\title{
Co-relation between waist circumference and serum triglyceride levels
}

\author{
Edwina Raj ${ }^{1, *}$, Umme Kulsum ${ }^{2}$, Apoorva ${ }^{3}$, Andalip ${ }^{4}$ \\ ${ }^{\mathbf{1}}$ Senior Dietitian, ${ }^{\mathbf{2}}$ Dietitian, ${ }^{3}$ Physician, ${ }^{4}$ Trainee, ${ }^{1}$ Dept. of Clinical and Nutrition, ${ }^{\mathbf{1 - 4}}$ Aster CMI Hospital Bangalore, Karnataka, \\ India
}

*Corresponding Author:

Email: edwina.raj@asterhospital.com

\begin{abstract}
The study aims at evaluating patients visiting for regular health check up presented with hypertriglyceridemia and positively influenced abdominal obesity. Hypertriglyceridaemia, a condition in which TG levels are elevated, is a common type of dyslipidaemia. It is believed to be a risk for coronary artery disease, diabetes and etiology for acute pancreatitis. Study was conducted on 30 patients who had high triglycerides and waist circumference were checked on their visit. Other anthropometry details were also recorded. As per the study the results showed a significant association between the Triglyceride levels and the waist circumference. The standard WHO cut off value for waist circumference were referred to compare the recorded values. A complete diet and lifestyle modification with patient education was reinforced to prevent triglyceride induced pancreatitis, insulin resistance and to reduce the risk for coronary heart disease among our health check patients.
\end{abstract}

Keywords: Triglycerides, Hypertriglyceridemia, Waist circumference, Dyslipidemia, Pancreatitis.

\section{Introduction}

Hypertriglyceridaemia, a condition in which triglyceride levels are elevated and a common type of dyslipidaemia. Abdominal obesity is a growing clinical and public health problem (Balkau et al. 2007). Some evidence indicated that waist circumference (WC) remains a significant predictor of chronic diseases such as type 2 diabetes, cardiovascular diseases, cancer, as well as total mortality after adjusting for body mass index (BMI) or fat mass (Bigaard et al. 2005). Also it's important whether measurement of waist circumference (WC) in addition to BMI is useful in primary care practice (Balkau et al. 2007). Triglyceride glucose-waist circumference, a novel and effective predictor of diabetes (Shuang Zheng 2016). The study was conducted to co-relate hyperglyceridemia and its impact on abdominal obesity together is a risk factor for chronic diseases. The excess fat waist circumference is a measurement taken around the abdomen at the level of the umbilicus (belly button). Waist circumference alone is not an individual parameter to indicate underlying medical condition but a marker to prevent the occurrence of illness. Adults require treatment for $\mathrm{TG}$ abnormalities with either lifestyle modification or the administration of medication. If intake of calories are more than expended through exercise, particularly triglycerides exceed due to excessive consumption of simple carbohydrates and hence it is important to counsel patients to modify and limit the intake of carbohydrate rich foods. Our body changes extra calories into triglycerides, and stores them in fat cells. When body needs energy it releases the triglycerides. Triglycerides and waist circumference--can identify patients with intra-abdominal obesity who have an increased risk of coronary artery disease and can add value in detecting risk of heart disease.

\section{Materials and Methods}

Patients with dyslipidemia were included in the study. Anthropometric assessment (weight, height and waist circumference) were recorded, Body Mass Index (BMI) was calculated and lipid measurements (serum total cholesterol LDL-cholesterol, HDL-cholesterol and triglycerides) were collected. Analyses of covariance were used to evaluate possible associations between levels of hypertriglyceridemia and waist circumference. The data were analyzed using cutoffs points for Asians ( $90 \mathrm{~cm}$ in men and $80 \mathrm{~cm}$ in women).

The aim of our study is to investigate the association between the hypertriglyceridemic waist phenotype amongst patients who have dyslipidemia.

\section{Results and Discussion}

30 participants were included. Mean Total cholesterol level was 180.37 Mean Triglyceride level was 182.75 . Mean waist circumference is $99.44 \mathrm{~cm}$.It is also seen that out of 30 individuals 18 individuals had a waist circumference greater than normal range (malesgreater than $102 \mathrm{Cm}$ Females- greater than $88 \mathrm{Cm}$ ). Out of these 19 individuals 14 individuals had increased serum triglyceride levels. 11 individuals had waist circumference within normal range out of which only 1 individual had high serum triglyceride levels.

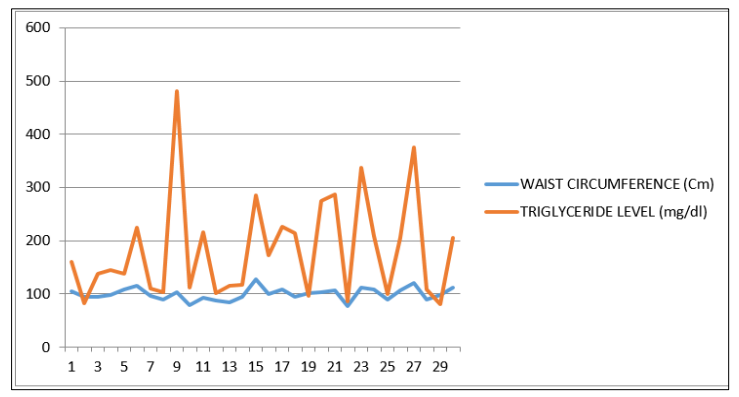

Fig. 1 


\section{Conclusion}

The study shows that individuals with greater waist circumference have increased triglyceride levels. Thus there is a significant association between waist circumference and Hypertriglyceridemia. Further study on the same with large sample size is needed.

\section{References}

1. Allison H Christian, EdD, Heidi Mochari. "Waist Circumference, Body Mass Index, and Their Association with Cardio metabolic and Global Risk" $J$ of PMC. 2009;12-19.

2. Benoit J. Arsenault, Isabelle Lemieux. "The hypertriglyceridemic-waist phenotype and the risk of coronary artery disease: results from the EPIC-Norfolk Prospective Population Study" CMAJ. 2010;1427-32

3. Weschenfelder, Camila; Marcadenti, Aline; Stein, Airton Tetelbom and Gottschall, Catarina Bertaso
Andreatta, "There is an association between serum triglycerides and waist circumference" Sao Paulo Medical Journal.

4. M. R. Fortson, S. N. Freedman, and P. D. Webster, "3rd. Clinical assessment of hyperlipidemic pancreatitis," The Ame J of Gastroent. 1995;90:213439. View at Google Scholar

5. The relationship between waist circumference and macronutrient intake in patients with type 2 diabetes in Tehran Article (PDF Available) in International Journal of Sciences: Basic and Applied Research (IJSBAR). 2013;5(8).

6. Triglyceride glucose waist circumference, A novel and effective predictor of diabetes in first-degree relatives of type 2 diabetes patients: cross-sectional and prospective cohort study Published online 2016 Sep doi: [10.1186/s12967-016-1020-8].

7. Geneva: World Health Organization. World Health Organization (WHO). Waist Circumference and WaistHip Ratio. Report of WHO Expert Consultation. 2008. 